\title{
Coloring the cube with rainbow cycles
}

\author{
Dhruv Mubayi* \\ Department of Mathematics, Statistics and Computer Science \\ University of Illinois \\ Chicago, IL 60607 U.S.A. \\ mubayi@uic.edu \\ Randall Stading \\ Department of Mathematics, Statistics and Computer Science \\ University of Illinois \\ Chicago, IL 60607, U.S.A. \\ rstadi1@uic.edu
}

Submitted: Dec 7, 2012; Accepted: Mar 27, 2013; Published: Apr 9, 2013

Mathematics Subject Classifications: 05C15, 05C35, 05C38, 05C55

\begin{abstract}
For every even positive integer $k \geqslant 4$ let $f(n, k)$ denote the minimim number of colors required to color the edges of the $n$-dimensional cube $Q_{n}$, so that the edges of every copy of the $k$-cycle $C_{k}$ receive $k$ distinct colors. Faudree, Gyárfás, Lesniak and Schelp proved that $f(n, 4)=n$ for $n=4$ or $n>5$. We consider larger $k$ and prove that if $k \equiv 0(\bmod 4)$, then there are positive constants $c_{1}, c_{2}$ depending only on $k$ such that

$$
c_{1} n^{k / 4}<f(n, k)<c_{2} n^{k / 4} .
$$

Our upper bound uses an old construction of Bose and Chowla of generalized Sidon sets. For $k \equiv 2(\bmod 4)$, the situation seems more complicated. For the smallest case $k=6$ we show that

$$
3 n-2 \leqslant f(n, 6)<n^{1+o(1)}
$$

with the lower bound holding for $n \geqslant 3$. The upper bound is obtained from Behrend's construction of a subset of integers with no three term arithmetic progression.
\end{abstract}

\footnotetext{
${ }^{*}$ Supported in part by NSF grant DMS 0969092.
} 


\section{Introduction}

Given graphs $G$ and $H$, and an integer $q \leqslant|E(H)|$, a $(G, H, q)$-coloring is an edge-coloring of $G$ such that the edges of every copy of $H$ in $G$ receive at least $q$ colors. Let $f(G, H, q)$ be the minimum number of colors in a $(G, H, q)$-coloring. This general problem is hopeless in most cases, for example, when $G$ and $H$ are cliques, and $q=2$, determining it is equivalent to determining the multicolor Ramsey number $R_{k}(p)$ which is a longstanding open problem. There has been more success in determining $f(G, H, q)$ when $G$ and $H$ are not cliques or when $q>2$ (or both). Many Ramsey problems have received considerable attention when studied on the $n$-dimensional cube. The papers $[3,4]$ are examples where anti-Ramsey problems for subcubes in cubes and problems about monochromatic cycles in cubes are investigated. In [13], Offner found the exact value for the maximum number of colors for which it is possible to edge color the hypercube so that all subcubes of dimension $d$ contain all colors. Related Turán type problems for subcubes in cubes have been studied in [2].

Rainbow cycles have also been well studied as subgraphs of $K_{n}$. Erdős, Simonovits and Sós [9] introduced $A R(n, H)$, the maximum number of colors in an edge coloring of $K_{n}$ such that it contains no rainbow copy of $H$, and provided a conjecture when $H$ is a cycle and showed that their conjecture was true when $H=C_{3}$. Alon [1] proved their conjecture for cycles of length four and Montellano-Ballesteros and Neumann-Lara [12] proved the conjecture for all cycles. More recently, Choi [7] gave a shorter proof of the conjecture.

We continue this theme in the current note and let $G=Q_{n}$, the $n$-dimensional cube, and $H=C_{k}$, the cycle of length $k$. Our focus is on $q=|E(H)|$, in which case we will call a $(G, H, q)$-coloring an $H$-rainbow coloring, assuming that $G$ is obvious from context (in this paper $G=Q_{n}$ always).

Definition 1. Fix $4 \leqslant k \leqslant 2^{n}$. Let $f(n, k)(g(n, k))$ be the minimum number of colors in a $C_{k}$-rainbow ( $Q_{k}$-rainbow) coloring of $Q_{n}$.

The smallest case $f(n, 4)$ was studied by Faudree, Gyárfás, Lesniak and Schelp [10] who proved that the trivial lower bound of $n$ is tight by providing, for all $n \geqslant 6$, a $C_{4}$-rainbow coloring with $n$ colors. We consider larger $k$. Our first result determines the order of magnitude of $f(n, k)$ for $k \equiv 0(\bmod 4)$.

Theorem 2. Fix a positive $k \equiv 0(\bmod 4)$. There are constants $c_{1}, c_{2}>0$ depending only on $k$ such that

$$
c_{1} n^{k / 4}<f(n, k)<c_{2} n^{k / 4}
$$

The case $k \equiv 2(\bmod 4)$ seems more complicated. Our results imply that for such fixed $k$ there are positive constants $c_{1}^{\prime}, c_{2}^{\prime}$ with

$$
c_{1}^{\prime} n^{\lfloor k / 4\rfloor}<f(n, k)<c_{2}^{\prime} n^{\lceil k / 4\rceil} .
$$


We believe that the lower bound is closer to the truth. As evidence for this, we tackle the smallest case in this range, $k=6$. As we will observe later, the lower bound $f(n, 6) \geqslant$ $3 n-2$ is easy to see for $n \geqslant 3$, and we obtain the following upper bound.

Theorem 3. For every $\epsilon>0$ there exists $n_{0}$ such that for $n>n_{0}$

$$
3 n-2 \leqslant f(n, 6)<n^{1+\epsilon}
$$

with the lower bound holding for all $n \geqslant 3$.

It is rather easy to see that $g(n, 3)=f(n, 6)$. Indeed, if $Q_{n}$ is edge-colored so that every $Q_{3}$ is rainbow, then every $C_{6}$ is rainbow since each one is contained in a rainbow $Q_{3}$ and so $g(n, 3) \geqslant f(n, 6)$. On the other hand, it is easy to see that any two edges of a $Q_{3}$ lie in some $C_{6}$ and therefore if $Q_{n}$ is edge-colored so that every $C_{6}$ is rainbow then every $Q_{3}$ must also be rainbow and so $g(n, 3) \leqslant f(n, 6)$.

Since $C_{4}=Q_{2}$, the following corollary can also be considered an analogue of the result [10] to subcubes.

Corollary 4. As $n \rightarrow \infty$, we have $g(n, 3)=n^{1+o(1)}$.

We will consider the vertices of $Q_{n}$ as binary vectors of length $n$ or as subsets of $[n]=$ $\{1, \ldots, n\}$, depending on the context (with the natural bijection $\vec{v} \leftrightarrow v$ where $\vec{v}$ is the incidence vector for $v \subset[n]$, i.e. $\vec{v}_{i}=1$ iff $\left.i \in v\right)$. In particular, whenever we write $v-w$ we mean set theoretic difference, $v \cup w$ or $v \cap w$ we mean set union/intersection and when we write $\vec{v} \pm \vec{w}$ we mean vector addition/subtraction modulo 2 . We write $e_{i}$ for the standard basis vector, so $e_{i}$ is one in the $i$ th coordinate and zero in all other coordinates. Given an edge $f=u v$ of $Q_{n}$ where $\vec{v}=\vec{u}+e_{s}$ for some $s$, we say that $v$ is the top vertex of $f$ and $u$ is the bottom vertex. We will say the an edge is on level $i$ of $Q_{n}$ if its bottom vertex corresponds to a vector with $i-1$ ones and the top vertex to a vector with $i$ ones.

\section{Proof of Theorem 2}

The lower bound in Theorem 2 follows from the easy observation that in a $C_{k}$-rainbow coloring all edges at level $k / 4$ must receive distinct colors. Indeed, given any two such edges $f_{1}=v w$ and $f_{2}=x y$, where $\vec{w}=\vec{v}+e_{i}$ and $\vec{y}=\vec{x}+e_{j}$, it suffices to find a copy of $C_{k}$ containing $f_{1}$ and $f_{2}$. If $f_{1}$ and $f_{2}$ are incident then it is clear that we can find a $C_{k}$ containing them as long as $n>k$ which we may clearly assume. The two cases are illustrated below where $r=k / 2-2$ and $s_{i} \notin w \cup y$ for all $i \in\{1, \ldots, r\}$. 

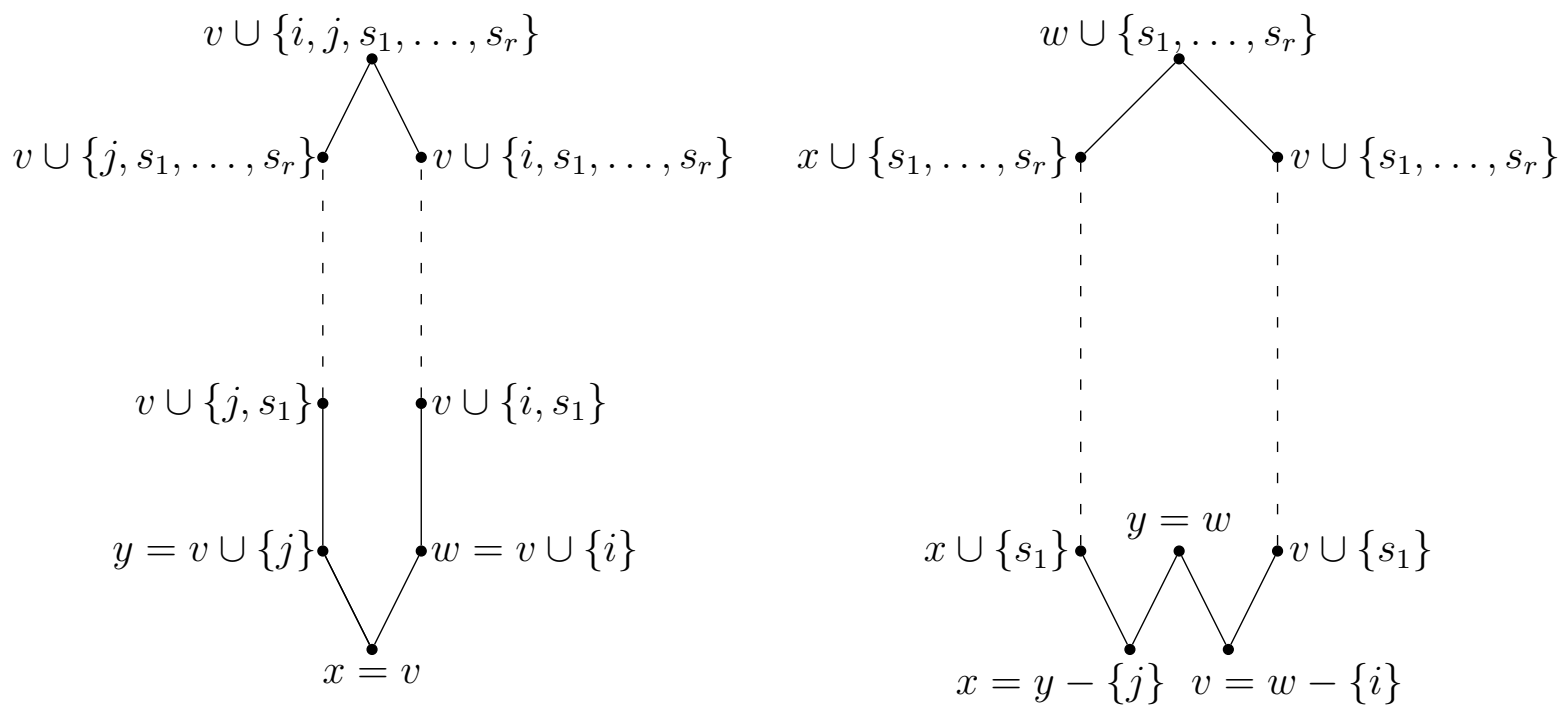

Now, suppose $f_{1}$ and $f_{2}$ are not incident. We know that $|x \Delta v| \leqslant k / 2-2$ since $x$ and $v$ are each sets of size $k / 4-1$. By successively deleting elements of $v$ and $x$ in the appropriate order, we can obtain a $v, x$-path of length $k / 2-2$. Then, since $w$ and $y$ are sets of size $k / 4$, we may find a $w, y$-path of length $k / 2$ between them by successively adding the elements of $y$ to $w$ and vice versa along with extra elements as needed. The two paths along with the edges $v w$ and $x y$ form a cycle of length $k$. This is shown in the following diagram. Let $y-w=\left\{y_{1}, \ldots, y_{m}\right\}, w-y=\left\{w_{1}, \ldots, w_{m}\right\}$ and $w \cap y=\left\{z_{1}, \ldots, z_{l}\right\}$ where $m+l=k / 4$. Let $\left\{s_{1}, \ldots, s_{r}\right\}$ again be a set such that $s_{i} \notin y \cup w$ with $r=k / 4-m$.

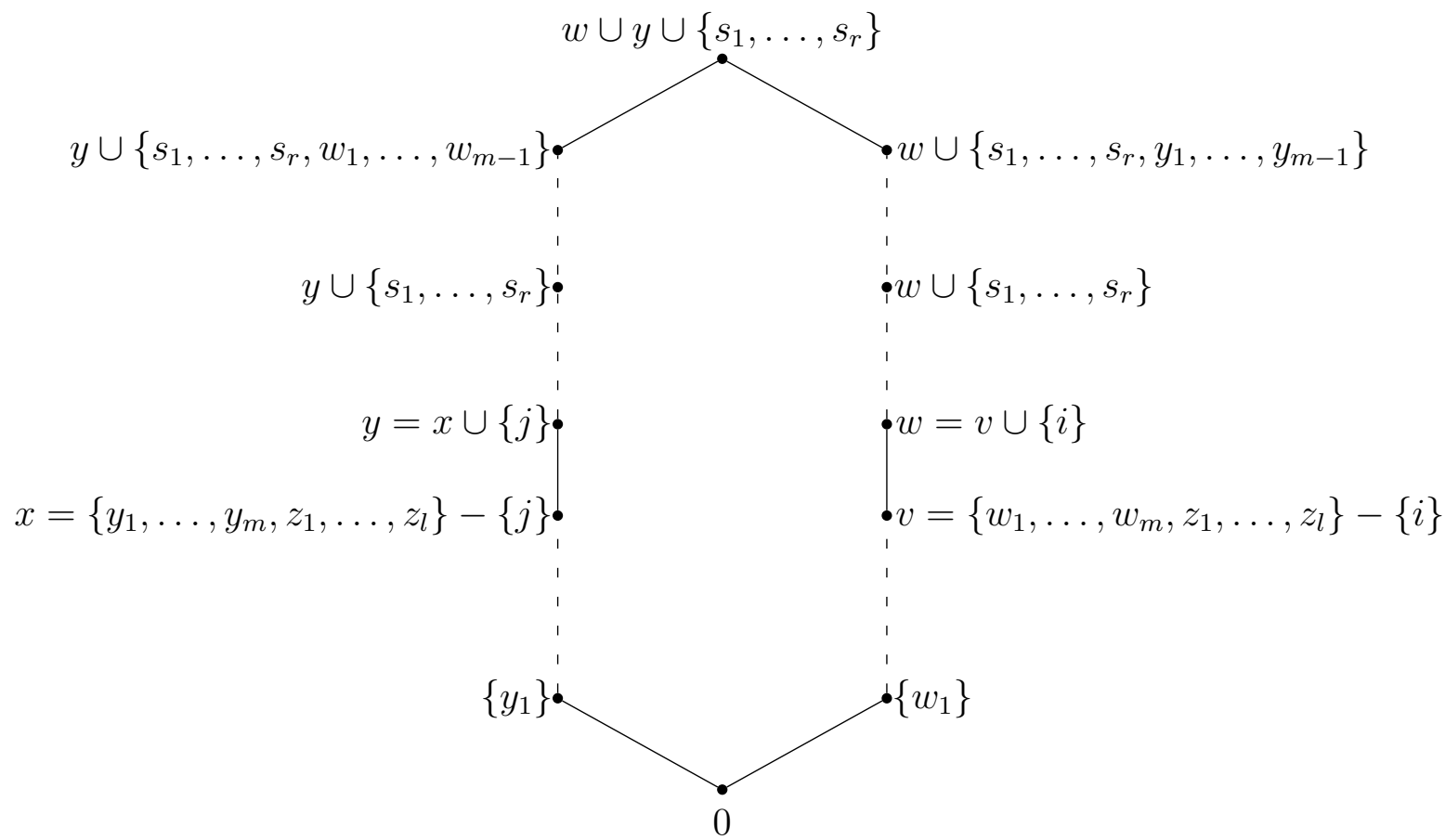


For the upper bound we need a classical construction of generalized Sidon sets by Bose and Chowla. A $B_{t}$-set $S=\left\{s_{1}, \ldots, s_{n}\right\}$ is a set of integers such that if $1 \leqslant i_{1} \leqslant i_{2} \leqslant \cdots \leqslant$ $i_{t} \leqslant n$ and $1 \leqslant j_{1} \leqslant j_{2} \leqslant \cdots \leqslant j_{t} \leqslant n$, then

$$
s_{i_{1}}+\cdots+s_{i_{t}} \neq s_{j_{1}}+\cdots+s_{j_{t}}
$$

unless $\left(i_{1}, \ldots, i_{t}\right)=\left(j_{1}, \ldots, j_{t}\right)$. A consequence of this is that if $P, Q$ are nonempty disjoint subsets of $[n]$ with $|P|=|Q| \leqslant t$, then

$$
\sum_{i \in P} s_{i} \neq \sum_{j \in Q} s_{j}
$$

The result below is phrased in a form that is suitable for our use later.

Theorem 5. (Bose-Chowla [6]) For each fixed $t \geqslant 2$, there is a constant $A>1$ such that for all $n$, there is a $B_{t}$-set $S=\left\{s_{1}, \ldots, s_{n}\right\} \subset\left\{1,2, \ldots,\left\lfloor A n^{t}\right\rfloor\right\}$.

Now we provide the upper bound construction for Theorem 2.

Construction 1. Let $t=k / 4-1$ and $S=\left\{s_{1}, \ldots, s_{n}\right\} \subset\left\{1,2, \ldots,\left\lfloor A n^{t}\right\rfloor\right\}$ be a $B_{t^{-}}$set as above. For each $v \in V\left(Q_{n}\right)$, let

$$
a(v)=\sum_{i=1}^{n} \vec{v}_{i} s_{i}=\sum_{i: \vec{v}_{i}=1} s_{i} .
$$

Given $v w \in E\left(Q_{n}\right)$ with $\vec{w}=\vec{v}+e_{j}$, let $M=\left\lceil k A n^{t}\right\rceil$, and let

$$
d(v w)=a(v)+M j .
$$

Suppose further that $v w$ is at level $p$ and $p^{\prime}$ is the congruence class of $p$ modulo $k / 2$. Then the color of the edge $v w$ is

$$
\chi(v w)=\left(d(v w), p^{\prime}\right) .
$$

Let us now argue that this construction yields the upper bound in Theorem 2 .

Proof. First, the number of colors is at most

$$
\max _{v w} d(v w) \times \frac{k}{2} \leqslant\left(n \cdot \max s_{i}+M n\right) \frac{k}{2} \leqslant \frac{n k}{2} A n^{t}+\frac{n k}{2} M<k^{2} A n^{t+1}=k^{2} A n^{k / 4}
$$

as desired. Now we show that this is a $C_{k}$-rainbow coloring. Suppose for contradiction that $H$ is a copy of $C_{k}$ in $Q_{n}$ and $f_{1}=v w, f_{2}=x y$ are distinct edges of $H$ with $\chi\left(f_{1}\right)=\chi\left(f_{2}\right)$. Since $H$ spans at most $k / 2$ levels, $f_{1}$ and $f_{2}$ cannot lie in levels that differ by more than $k / 2$, so $\chi\left(f_{1}\right) \neq \chi\left(f_{2}\right)$ unless $f_{1}$ and $f_{2}$ are in the same level which we may henceforth assume. Let $v, x$ be the bottom vertices of $f_{1}, f_{2}$, and $\vec{w}=\vec{v}+e_{i}, \vec{y}=\vec{x}+e_{j}$. Assume without loss of generality that $i \leqslant j$. If $v=x$, then

$$
a(v)+M i=d(v w)=d(x y)=a(x)+M j=a(v)+M j .
$$


This implies that $i=j$ and contradicts the fact that $f_{1} \neq f_{2}$. We may therefore assume that $v \neq x$. Similarly, if $w=y$, then $i<j$ and

$a(w)-s_{i}+M i=a(v)+M i=d(v w)=d(x y)=a(x)+M j=a(y)-s_{j}+M j=a(w)-s_{j}+M j$.

This implies the contradiction $s_{j}-s_{i}=M(j-i) \geqslant M>A n^{t}>s_{j}-s_{i}$. Consequently, we may assume that $v w$ and $x y$ share no vertex. If $|v \Delta x|>k / 2$, then any $v, x$-path in $Q_{n}$ has length more than $k / 2$ so there can be no cycle of length $k$ containing both $v$ and $x$, contradiction. So we may assume that $|v \triangle x| \leqslant k / 2$. Now $\chi(v w)=\chi(x y)$ implies that

$$
a(v)+M i=d(v w)=d(x y)=a(x)+M j
$$

and this yields

$$
M(j-i)=M j-M i=a(v)-a(x)=a(v-x)-a(x-v) \leqslant \frac{|v \triangle x|}{2} A n^{t} \leqslant \frac{k}{4} A n^{t}<M .
$$

Consequently, we may assume that $i=j, a(v)=a(x), a(v-x)=a(x-v)$ and $|v \triangle x|=$ $|w \triangle y|$. If $|v-x|=|x-v| \leqslant k / 4-1$, then

$$
a(v-x)=\sum_{i \in v-x} s_{i} \neq \sum_{j \in x-v} s_{j}=a(x-v)
$$

due to (1), the definition of $S$ and $t=k / 4-1$. So we may assume that $|v-x|=$ $|x-v|=k / 4$ and $|w \triangle y|=|v \triangle x|=k / 2$. This implies that $\operatorname{dist}_{Q_{n}}(w, y)=\operatorname{dist}_{Q_{n}}(v, x)=$ $k / 2$. Together with edges $f_{1}, f_{2}$, we conclude that $C$ must have at least $k+2$ edges, contradiction.

\section{Proof of Theorem 3}

We will first show the lower bound $f(n, 6) \geqslant 3 n-2$ for $n \geqslant 3$ by providing a set of edges $F$ of size $3 n-2$ which must all receive distinct colors in a $C_{6}$-rainbow coloring of $Q_{n}$. Fix an edge $f$ on the first level of $Q_{n}$. For each copy $C$ of $C_{4}$ containing $f$, let $F_{C}$ be the set of four edges in $C$. Let $F=\cup_{C} F_{C}$, where the union is taken over all copies $C$ of a $C_{4}$ containing $f$. There are $n-1$ such $C$ 's and any two distinct $C$ 's share at most one edge, so $|F|=3(n-1)+1=3 n-2$.

Now, suppose $f_{1}, f_{2} \in F$ receive the same color. If $f_{1}$ and $f_{2}$ lie on the same $C_{4}$, then let $h$ be one of the two remaining edges of that $C_{4}$ and take a different $C_{4}$ which contains $h$. Delete $h$ and observe that the remaining six edges of the two $C_{4}$ 's form a $C_{6}$. If $f_{1}$ and $f_{2}$ do not lie on the same $C_{4}$, then their $C_{4}$ 's share the edge $f$. Now delete $f$ and observe that the remaining six edges form a $C_{6}$. We conclude that all the edges in $F$ must receive distinct colors. 
To obtain the upper bound, we will give an explicit coloring that makes use of a classical construction of Behrend on sets of integers with no arithmetic progression of size three. Let $r_{3}(N)$ denote the maximum size of a subset of $\{1, \ldots, N\}$ that contains no 3 -term arithmetic progression.

Theorem 6. (Behrend [5]) There is a $c>0$ such that if $N$ is sufficiently large, then

$$
r_{3}(N)>N^{1-\frac{c}{\sqrt{\log N}}}
$$

Theorem 6 clearly implies that for $\epsilon>0$ and sufficiently large $N$ we have $r_{3}(N)>N^{1-\epsilon}$. The error term $\epsilon$ was improved recently by Elkin [8] (see [11] for a simpler proof) and using Elkin's result would give corresponding improvements in our result.

Construction 2. Let $\epsilon>0$ and $n$ be sufficiently large. Put $N=\left\lceil n^{1+\epsilon}\right\rceil$ and let $S=\left\{s_{1}, \ldots, s_{n}\right\} \subset\{1, \ldots, N\}$ contain no 3 -term arithmetic progression. Such a set exists by Theorem 6 since

$$
n>n^{1-\epsilon^{2}}=n^{(1-\epsilon)(1+\epsilon)}>N^{1-2 \epsilon} .
$$

Let

$$
a(v)=\sum_{i=1}^{n} \vec{v}_{i} s_{i} .
$$

Consider the edge $v w$, where $\vec{w}=\vec{v}+e_{k}$. Let

$$
d(v w)=a(v)+2 s_{k} \in Z_{2 N} .
$$

We emphasize here that we are computing $d(v w)$ modulo $2 N$. Suppose further that $v w$ is at level $p$ and $p^{\prime}$ is the congruence class of $p$ modulo 3. Then the color of the edge $v w$ is

$$
\chi(v w)=\left(d(v w), p^{\prime}\right)
$$

The number of colors used is at most $6 N<n^{1+2 \epsilon}$ as required.

Proof. We will now show that this is a $C_{6}$-rainbow coloring. Due to the second coordinate, it suffices to show that any two edges $f_{1}, f_{2}$ of a $C_{6}$ which are on the same of level of $Q_{n}$ receive different colors. If $f_{1}$ and $f_{2}$ are incident, then they meet either at their top vertices or bottom vertices. If incident at their bottom vertices, the edges are colored as follows and thus are distinctly colored:

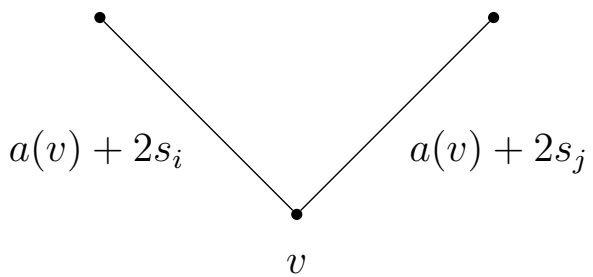

If incident at their top vertices, the edges lie on a $C_{4}$ and are therefore distinctly colored 


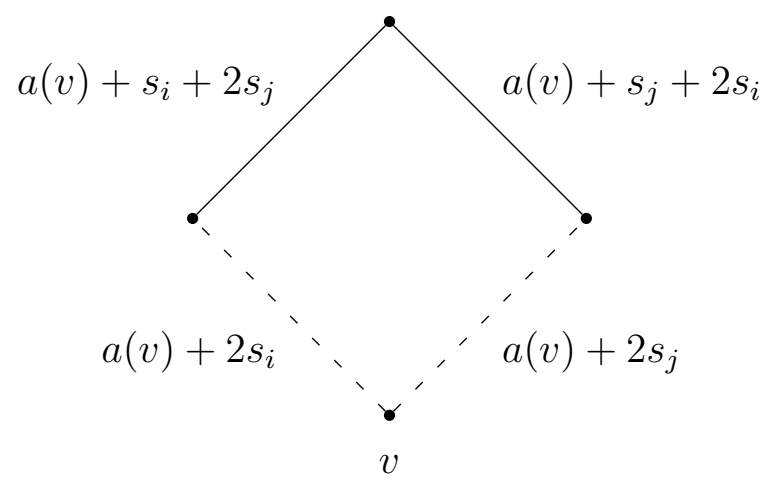

If $f_{1}$ and $f_{2}$ are not incident, then there must be a path of length two between their bottom vertices. For if not, then they could not lie on a $C_{6}$ as the shortest path between their top vertices has length at least two. Moreover, the top vertices of $f_{1}$ and $f_{2}$ have symmetric difference precisely two since there is a path of length two between them. With these conditions, there are three ways the edges may be colored.
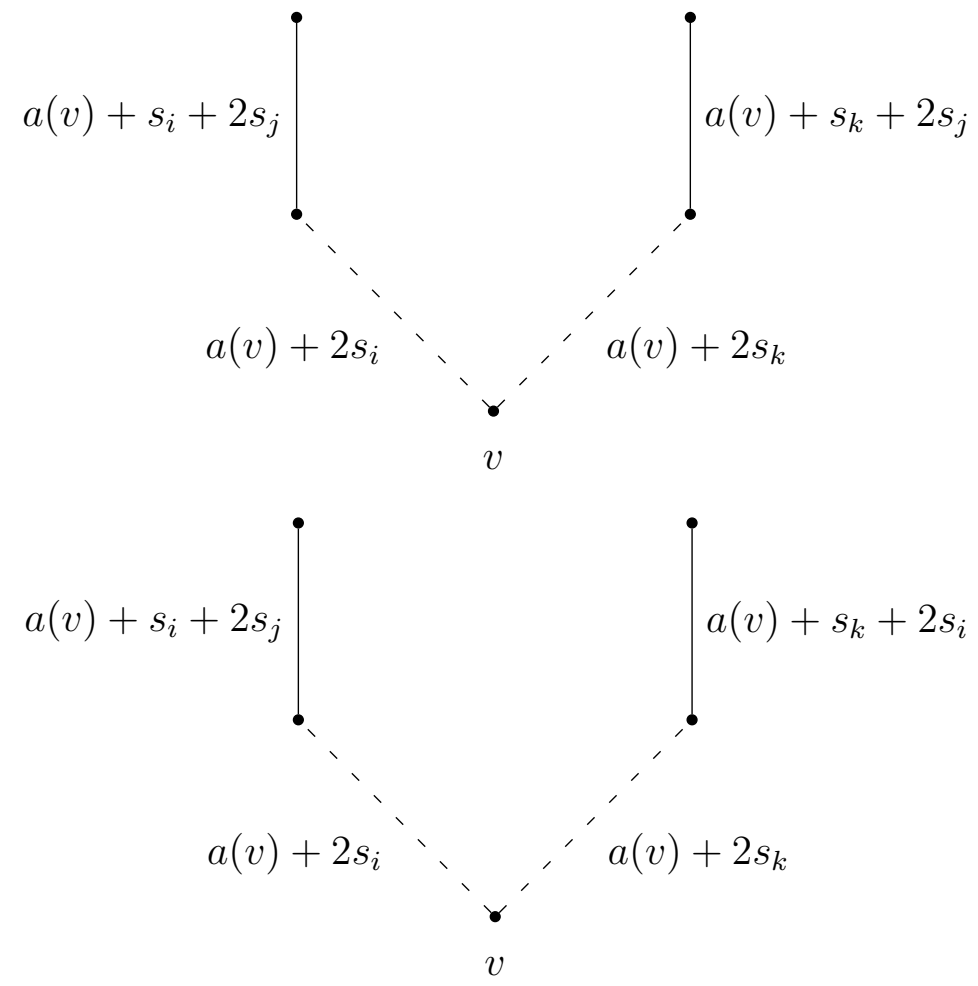


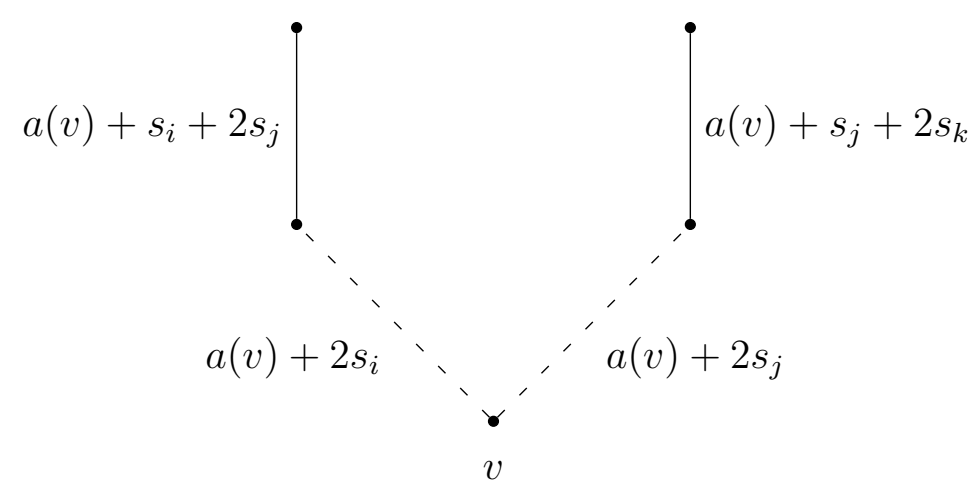

In the first coloring, $s_{i}+2 s_{j} \neq s_{k}+2 s_{j}$ holds due to $i$ and $k$ being distinct. In the second and third colorings, $s_{i}+2 s_{j} \neq s_{k}+2 s_{i}$ and $s_{i}+2 s_{j} \neq s_{j}+2 s_{k}$ hold due to our set $S$ being free of three term arithmetic progressions.

\section{Concluding Remark}

Our results imply a tight connection between $C_{k}$-rainbow colorings in the cube and constructions of large generalized Sidon sets. When $k \equiv 0(\bmod 4)$ Construction 1 gives the correct order of magnitude, however for $k \equiv 2(\bmod 4)$ the same method does not work. In this case an approach similar to Construction 2 would work provided we can construct large sets that do not contains solutions to certain equations.

Conjecture 7. Fix $4 \leqslant k \equiv 2(\bmod 4)$. Then $f(n, k)=n^{\lfloor k / 4\rfloor+o(1)}$.

For the first open case $k=10$, we can show that $f(n, 10)=n^{2+o(1)}$ provided one can construct a set $S \subset[N]$ with $|S|>N^{1 / 2-o(1)}$ that contains no nontrivial solution to any of the following equations:

$$
\begin{aligned}
x_{1}+x_{2} & =x_{3}+x_{4} \\
x_{1}+x_{2}+x_{3} & =x_{4}+2 x_{5} \\
x_{1}+2 x_{2} & =x_{3}+2 x_{4} .
\end{aligned}
$$

Ruzsa $[14,15]$ defined the genus $g(E)$ of an equation

$$
E: \quad a_{1} x_{1}+\cdots+a_{k} x_{k}=0
$$

as the largest $m$ such that there is a partition $S_{1} \cup \ldots \cup S_{m}$ of $[k]$ where the $S_{i}$ are disjoint, non-empty and for all $j$,

$$
\sum_{i \in S_{j}} a_{i}=0
$$

A solution $\left(x_{1}, \ldots, x_{k}\right)$ of $E$ is trivial if there are $l$ distinct numbers among $\left\{x_{1}, \ldots, x_{k}\right\}$ and (2) holds for a partition $S_{1} \cup \ldots \cup S_{l}$ of $[k]$ into disjoint, non-empty parts such that $x_{i}=x_{j}$ if and only if $i, j \in S_{v}$ for some $v$. Ruzsa showed that if $S \subset[n]$ has no 
nontrivial solutions to $E$ then $|S| \leqslant O\left(n^{1 / g(E)}\right)$. The question of whether there exists $S$ with $|S|=n^{1 / g(E)-o(1)}$ remains open for most equations $E$. The set of equations above has genus two so it is plausible that one can prove Conjecture 7 for $k=10$ using this approach. For the general case, we can provide a rainbow coloring if our set $S$ contains no nontrivial solutions to any of the three equations below with $m=\lfloor k / 4\rfloor$.

$$
\begin{aligned}
x_{1}+\cdots+x_{m} & =x_{m+1}+\cdots+x_{2 m} \\
x_{1}+\cdots+x_{m}+x_{m+1} & =x_{m+2}+\cdots+x_{2 m}+2 x_{2 m+1} \\
x_{1}+\cdots+x_{m-1}+2 x_{m} & =x_{m+1}+\cdots+x_{2 m-1}+2 x_{2 m} .
\end{aligned}
$$

The set of equations above has genus $m=\lfloor k / 4\rfloor$, so if Ruzsa's question has a positive answer, then we would be able to construct a set of the desired size.

\section{Acknowledgements}

The authors would like to thank the referee for the careful reading and commentary, particularly for pointing out the improved lower bound for $f(n, 6)$.

\section{References}

[1] N. Alon, On a conjecture of Erdős, Simonovits, and Sós concerning anti-Ramsey theorems. J. Graph Theory 7 (1983) no. 1, 91-94.

[2] N. Alon, A. Krech, T. Szabó, Turán's theorem in the hypercube. SIAM J. Discrete Math. 21 (2007), no. 1, 66-72.

[3] N. Alon, R. Radoičić, B. Sudakov and J. Vondrák, A Ramsey-type result for the hypercube. J. Graph Theory 53 (2006), no. 3, 196-208.

[4] M. Axenovich, H. Harborth, M. Möller and I. Schiermeyer, Rainbows in the hypercube. (English summary) Graphs Combin. 23 (2007), no. 2, 123-133.

[5] F. A. Behrend, On sets of integers which contain no three terms in arithmetical progression. Proc. Nat. Acad. Sci. U.S.A. 32, (1946). 331-332.

[6] R. C. Bose, S. Chowla, Theorems in the additive theory of numbers. Comment. Math. Helv. 37 1962/1963 141--147.

[7] J. Choi, A short proof of anti-Ramsey number for cycles. preprint.

[8] M. Elkin, An improved construction of progression-free sets. Israel J. Math. 184 (2011), 93-128.

[9] P. Erdős, M. Simonovits, and V. T. Sós, Anti-Ramsey theorems. Infinite and finite sets. Colloq. Math. Soc. Janos Bolyai 10 (1975) 633-643.

[10] R. J. Faudree, A. Gyárfás, L. Lesniak and R.H. Schlep, Rainbow coloring the cube. J. Graph Theory 17 (1993), no. 5, 607-612. 
[11] B. Green, J. Wolf, A note on Elkin's improvement of Behrend's construction, in Additive Number Theory (Festschrift in honour of Mel Nathanson), Springer 2010, $141-144$.

[12] J. J. Montellano-Ballesteros, V. Neumann-Lara, An anti-Ramsey theorem on cycles. Graphs Combin. 22 (2005) no. 3, 343-354.

[13] D. Offner, Polychromatic colorings of subcubes of the hypercube. SIAM J. Discrete Math 22 (2008) no. 2, 450--454.

[14] I. Z. Ruzsa, Solving a linear equation in a set of integers. I. Acta Arith. 65 (1993), no. 3, 259-282.

[15] I. Z. Ruzsa, Solving a linear equation in a set of integers. II. Acta Arith. 72 (1995), no. $4,385-397$. 